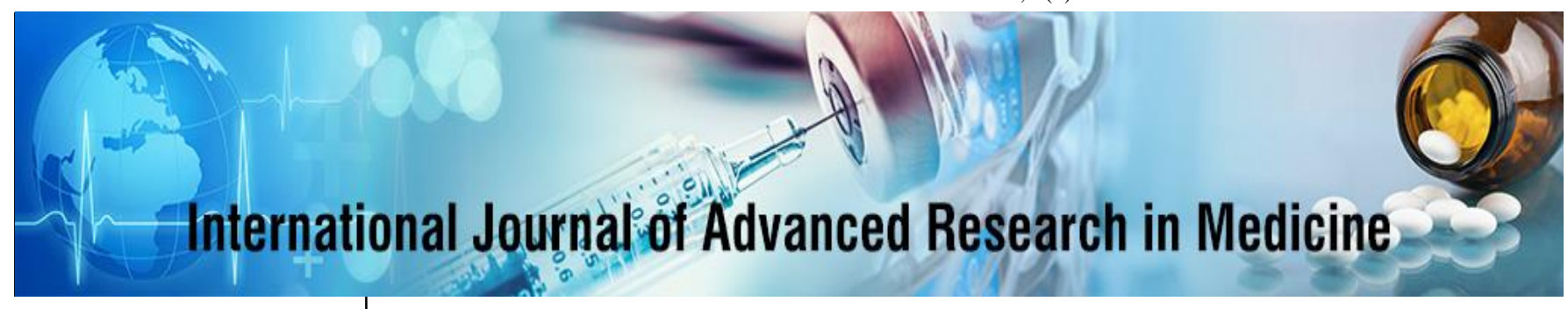

E-ISSN: 2706-9575

P-ISSN: 2706-9567

IJARM 2022; 4(1): 103-106

Received: 15-11-2021

Accepted: 18-12-2021

\section{Hemant Mehta}

Department of Nephrology,

Lilavati Hospital and

Research Centre, Mumbai,

Maharashtra, India

\section{Abhay Bhave}

Department of Hematology, Lilavati Hospital and Research Centre, Mumbai.

India

\section{Jhoomar Makhija}

Department of Nephrology, Lilavati Hospital and Research Centre, Mumbai, Maharashtra, India

Wasiyeeullah Shaikh Department of Nephrology, Lilavati Hospital and Research Centre, Mumbai, Maharashtra, India

Bhagyashree Gorakh Department of Nephrology, Lilavati Hospital and Research Centre, Mumbai, Maharashtra, India

\section{Pallavi Tanpure}

Department of Nephrology, Lilavati Hospital and Research Centre, Mumbai, Maharashtra, India

\section{Nawal Kaz}

Department of Hematology, Lilavati Hospital and Research Centre, Mumbai, India
Corresponding Author: Hemant Mehta

Department of Nephrology, Lilavati Hospital and Research Centre, Mumbai, Maharashtra, India

\section{Acute kidney injury due to deposition disease}

\author{
Hemant Mehta, Abhay Bhave, Jhoomar Makhija, Wasiyeeullah Shaikh, \\ Bhagyashree Gorakh, Pallavi Tanpure and Nawal Kaz
}

DOI: $\underline{\text { https://doi.org/10.22271/27069567.2022.v4.i1b.345 }}$

\begin{abstract}
Acute kidney injury presenting without obvious cause is a diagnostic dilemma. One condition to be considered is monoclonal gammopathy. A case of light chain deposition disease was detected as a cause, involving kidney and extra renal site. It is a part of monoclonal immunoglobulin deposition disease, and may or may not be related to multiple myeloma. Delayed diagnosis can lead to end stage kidney disease, requiring long term dialysis, with its vascular access problems, preventing the treatment by autologous stem cell transplant. The case is presented with review of literature.
\end{abstract}

Keywords: Acute kidney injury, chronic kidney disease, light chain deposition disease, monoclonal immunoglobulin deposition disease, dialysis catheter related atrial thrombus

\section{Introduction}

An accurate diagnosis of acute kidney injury (AKI), either as AKI or presenting with an underlying chronic kidney disease (CKD) (acute on CKD) sometimes causes a clinical dilemma, when obvious cause is 'unknown'. It needs a proper history, examination and investigations. One condition to be considered in differential diagnosis of AKI on CKD or AKI of 'unknown' etiology is monoclonal gammopathies, characterized by the monoclonal proliferation of the lymphoplasmacytic cells in the bone marrow and monoclonal immunoglobulin ( $\mathrm{Ig}$ ) deposition in the tissues (deposition diseases, DD). The most common ones include solitary plasmacytoma, monoclonal gammopathy of undetermined significance (MGUS), smoldering myeloma, multiple myeloma (MM), and immunoglobulin light chain amyloidosis (AL amyloidosis). Abnormal renal indices can be the first sign of multiple myeloma or light chain amyloidosis. Despite significant advances in the treatment of MM, the overall prognosis of renal recovery is still poor ${ }^{[1,2]}$. The renal injury can be $\mathrm{Ig}$ mediated, non-Ig mediated or glomerulonephritis (GN). Ig mediated injury is commonest, (cast nephropathy or myeloma kidney), monoclonal immunoglobulin deposition disease (MIDD), and light chain amyloidosis (AL). Non-Ig mediated or Ig-independent mechanisms include hypercalcemia, volume depletion, sepsis, tumor lysis, medication toxicity and plasma cell invasion of the renal parenchyma. GN can present as membranoproliferative, crescentic, or cryoglobulinemic vasculitis. Occasionally minimal change disease or membranous disease can also be seen ${ }^{[3]}$. When kidney involvement is seen in patients with MGUS, it is called monoclonal gammopathy of renal significance (MGRS).

\section{Learning objective}

AKI has variety of causes, sometimes obvious but at times, difficult to establish the cause at the onset of disease. One such patient is reported below who presented with AKI and the etiology was found to be due to light chain deposition disease (LCDD), a disease categorized in the family of MIDD in the World Health organization (WHO) Classification of Tumors of Hematopoietic and Lymphoid Tissues ${ }^{[4]}$. A delayed diagnosis can lead to end stage renal disease (ESRD) requiring hemodialysis (HD) and further problems, making patient unsuitable for autologous stem cell transplantation (ASCT). Relevant literature on LCDD is reviewed.

\section{Case}

A 53 years old male was referred to emergency department in May 2016 for urgent dialysis due to azotemia, hyperkalemia and metabolic acidosis. 
Past history was significant as having hypertension for 7 years, on irregular treatment with telmisartan. His coronary angiogram in February 2016 was reported as normal. At that time, his S. Creatinine was $1.0 \mathrm{mg} / \mathrm{dl}$, and urine showed $2+$ protein. 24 hours urine protein was $1600 \mathrm{mg} /$ day, was advised kidney biopsy but was not done. On admission, his reports showed normocytic normochromic anemia, with normal white cell count and platelets. Urinalysis showed 4+ proteinuria, with $10-12$ pus cells and 8-10 red cells. Spot urine for protein-creatinine ratio was $4.5 \mathrm{gm} / \mathrm{gm}$. S. Creatinine was $8.6 \mathrm{mg} / \mathrm{dl}$ and potassium was $6.7 \mathrm{mEq} / \mathrm{L}$. S. Calcium was $9.28 \mathrm{mg} / \mathrm{dl}$, phosphorus $6.6 \mathrm{mg} / \mathrm{dl}$, uric acid $15.4 \mathrm{mg} / \mathrm{dl}$, total proteins $6.6 \mathrm{gm} / \mathrm{dl}$ (albumin 3.4, globulin 3.2). S. LDH was $559 \mathrm{U} / \mathrm{L}, \mathrm{CPK} 221 \mathrm{IU} / \mathrm{L}$. Venous bicarbonate $\left(\mathrm{TCO}_{2}\right)$ was $11 \mathrm{mEq} / \mathrm{L}$, suggestive of metabolic acidosis. 2D Echo showed left ventricular ejection fraction of $40 \%$, with mild generalized hypokinesia and pulmonary artery systolic pressure of $43 \mathrm{~mm} \mathrm{Hg}$. Ultrasonography showed both kidneys to be $11 \mathrm{cms}$, with raised cortical echogenecity and maintained cortico medullary differentiation. It also showed bilateral pleural effusions. S. Parathyroid hormone was $117 \mathrm{pg} / \mathrm{ml}$.

He was initiated on HD. Further investigations showed $\mathrm{HbSAg}, \mathrm{HCV}, \mathrm{HIV}$ negative, normal Serum C3 \& C4 levels, ANA, Anti ds-DNA, ANCA negative. Serum protein electrophoresis did not show presence of $M$ band. Urine for Bence Jones proteins was negative. Immunofixation electrophoresis did not show presence of monoclonal gammopathy. Serum free light chain (FLC) assay showed kappa chain level of $876 \mathrm{mg} / \mathrm{L}$ (Normal 3.3 - $19.4 \mathrm{mg} / \mathrm{L}$ ); and lambda chain at $38.52 \mathrm{mg} / \mathrm{L}$ (Normal $5.7-26.3 \mathrm{mg} / \mathrm{L}$ ), giving $\mathrm{k} / \mathrm{l}$ ratio of 22.87 (Normal 0.26 to 1.65 ) (For patients with eGFR $<60 \mathrm{~mL} / \mathrm{min} / 1.73 \mathrm{~m}^{2}$, normal $\mathrm{k} / \mathrm{l}$ ratio is $0.37-$ 3.1). EMG-NC showed sensory motor axonal neuropathy in both distal lower limbs.

A bone marrow aspirate showed $4 \%$ plasma cells. Bone marrow biopsy and Fluorescence in situ hybridization (FISH) were also done. Though clinical diagnosis of multiple myeloma was suspected, bone marrow aspiration and biopsy did not confirm the same, reported as reactive marrow. PET-CT scan showed a hyper metabolic left suprarenal mass (Figure 1, 2). A CT guided biopsy of the mass was reported as pheochromocytoma or paraganglioma. Pheochromocytoma was unlikely in absence of severe or uncontrolled hypertension and this lesion could still not explain his renal failure. After stabilizing him with HD, a diagnostic test in the form of USG guided kidney biopsy was performed. Biopsy showed increase in mesangial matrix and acellular nodular deposits in 13/18 glomeruli, which were Congo red negative (Figure 3). Pink homogenous eosinophilic material deposition was seen around tubular and blood vessel walls (Congo red negative) (Figure 4). Kappa stain was positive in eosinophilic peritubular and perivascular deposits and in few glomeruli (Figure 5). The tissue from suprarenal mass stained positive for kappa stain in extracellular homogenous material (Figure 6 ). The FISH study on bone marrow showed large deletion of $13 \mathrm{q} /$ Monosomy 13 in $60 \%$ of cells, inferred by deletion of both $13 \mathrm{q} 14.3$ and $13 \mathrm{q} 34$ regions of which $4 \%$ also showed Monosomy 12.

A final diagnosis of LCDD due to kappa chain causing AKI and extra renal involvement of left supra renal area was made. He was started on chemotherapy with bortezomib, cyclophosphamide and dexamethasone (BCD) and lenalidomide. He continued to be dialysis dependent. After 1.5 years, his SFLC ratio normalized and he was offered ASCT. However, his HD dependency, and the problems related to his vascular access came in the way. He was on tunneled cuffed HD catheter in right internal jugular vein, (after failed arterio-venous fistula, AVF), which caused catheter related right atrial thrombus (CRAT), and did not respond to thrombolysis and oral anticoagulation. He refused mechanical (AngioVac device $($ ) or surgical thrombectomy and hence he was denied bone marrow transplant by hematologist, till the CRAT is cleared, as it could be a potential source of sepsis or pulmonary thromboembolism in future. Unfortunately he died due to Covid 19 infection in November 2020.

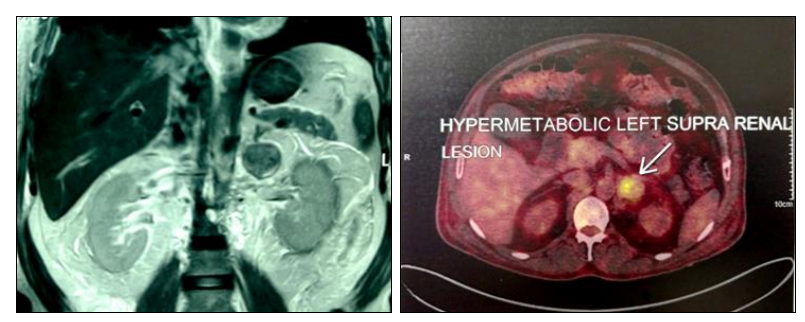

Fig 1, 2: PET-CT scan showing hypermetabolic left suprarenal mass

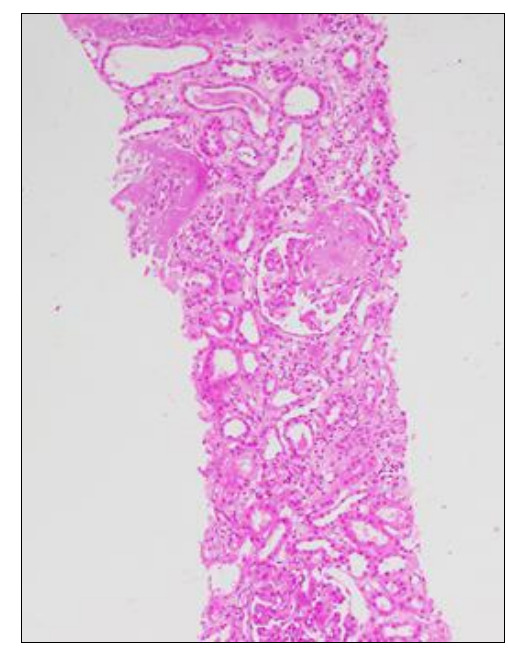

Fig 3: Kidney biopsy showed increase in mesangial matrix and acellular nodular deposits in 13/18 glomeruli, which were Congo red negative

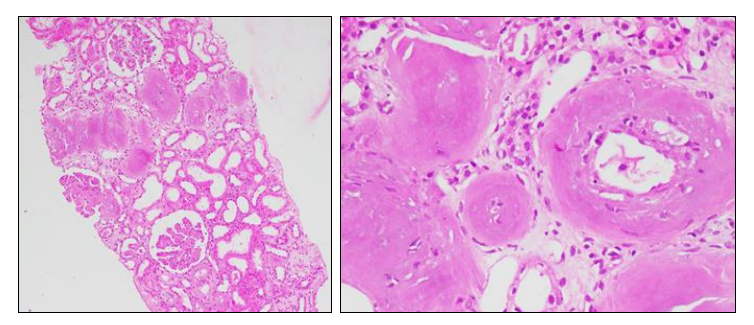

Fig 4: Pink homogenous eosinophilic material deposition was seen around tubular and blood vessel walls (congo red negative)

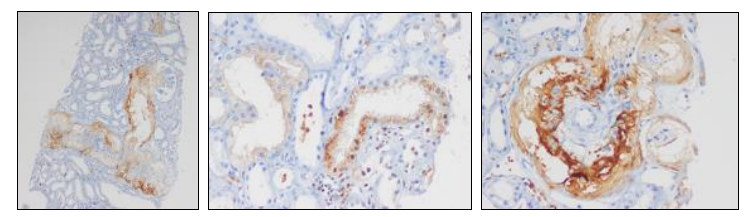

Fig 5: Kappa stain was positive in eosinophilic peritubular and perivascular deposits and in few glomeruli 


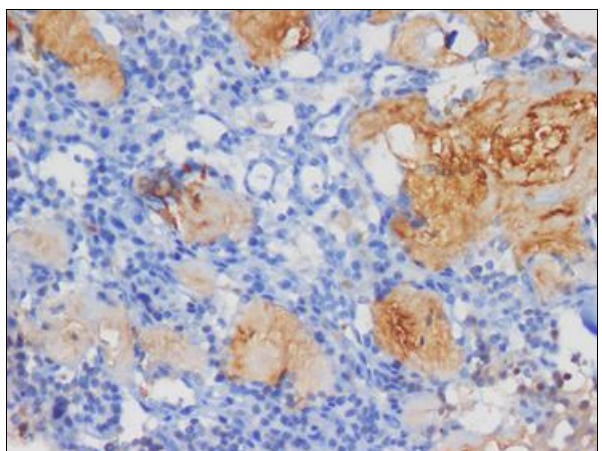

Fig 6: The tissue from suprarenal mass stained positive for kappa stain in extracellular homogenous material

\section{Discussion}

This case brings out 2 aspects of a disease, viz. unusual cause of AKI leading to end stage kidney failure (ESKD) requiring long term $\mathrm{HD}$ and vascular access problems, and LCDD. Though the presentation was of AKI, he had underlying CKD as he had proteinuria of $1600 \mathrm{mg} / \mathrm{day}, 4$ months prior to the presentation (the duration of which was not known), with normal S. Creatinine of $1.0 \mathrm{mg} / \mathrm{dl}$. So, he had underlying CKD (presumed glomerulonephritis, G1, A3; as per KDIGO CGA classification) ${ }^{[5]}$. Four months later, he presented with acute deterioration of renal function. There are multiple causes to be considered when such acute on CKD occurs, it needs simultaneous treatment and evaluation of cause. Causes of AKI like crescentic glomerulonephritis, thrombotic microangiopathy, drug related AKI (direct toxicity or crystalluria) and DD need quick work up to arrive at an early diagnosis, otherwise it may be too late to salvage the kidney function. Other rare causes of AKI include vascular causes, oxalate nephropathy, anticoagulation-related nephropathy and abdominal compartment syndrome.

\section{AKI - rare cause}

There was no clear-cut history, clinical finding, and investigation to get at a quick diagnosis. He needed urgent HD for control of his hyperkalemia and metabolic acidosis. The bone marrow for MM was negative. The final diagnosis of Light chain deposit disease LCDD) due to kappa chain and supra-renal involvement was unexpected. The incidence of LCDD is unknown. The median age at diagnosis is 58 years, and it is more common in men than in women. Approximately $50-60 \%$ of patients with LCDD have associated MM and $17 \%$ have MGUS or no evidence of neoplastic plasma cell proliferation ${ }^{[4]}$. The disease was very severe in progression, that it caused proteinuria detection to renal failure in 4 months. In spite of diagnosis and starting the chemotherapy, patient's kidney function did not improve and he became HD dependent. The treatment with BCD regimen resulted in complete remission of LCDD. The next step would have been to consider him for ASCT. However, his ongoing HD related issues never allowed him to settle and go ahead with final treatment for LCDD i.e. ASCT, which would probably have led to kidney transplant in future.

Problems of chronic hemodialysis: Difficult vascular access A reliable vascular access is essential for long term HD. Best access is AVF, created surgically. He had 2 such AVFs created, which failed to mature. On both occasions, balloon angioplasty of the juxta-anastomotic vein of AVF were unsuccessful. He became HD dependent with a tunneledcuff catheter (TCC) inserted through his right internal jugular vein. Several attempts to counsel him for $3^{\text {rd }}$ AVF failed. He had complications of TCC, one episode of catheter related blood stream infection (CRBSI), and later catheter related atrial thrombus (CRAT). Attempt to dissolve CRAT with alteplase (recombinant tissue plasminogen activator (rtPA) failed. He was then put on oral anticoagulation, which had to be discontinued as it was difficult to maintain target prothrombin time, due to HD and need for heparin. Hence, in consultation with hematologist, he was put on low molecular weight heparin on non-HD days, and regular heparin during dialysis on HD days. He was advised for CRAT clearance either surgically or through a mechanical device AngioVac $®$. He declined both. The CRAT clearance was essential prior to ASCT.

\section{MIDD - LCDD}

Monoclonal immunoglobulin deposition disease (MIDD) is a multi-system disease characterized by the deposition of monoclonal Ig molecules in various organs ${ }^{[6,7]}$. LCDD is the most common form of MIDD diagnosed, and it is a systemic disease. LCDD was first described in 1976 in two patients with end-stage renal disease as granular deposits of free light chains in multiple organs, including the kidneys that did not stain with Congo red ${ }^{[8]}$. Many organs are affected by the deposition of monotype immunoglobulin light chain (LCs), but the kidneys are always affected ${ }^{[8]}$. Because FLCs are rapidly cleared from the serum and are largely filtered by the kidneys, this organ is a prominent target for LC deposition and is often damaged. Extra renal involvement is primarily noted at autopsy and is usually confined to the perivascular regions of the affected organs. Extra renal manifestations were reported as cardiac (21\%), liver $(19 \%)$, cerebral infarction and hemorrhage, peripheral neuropathy and mononeuritis multiplex and adrenal insufficiency ${ }^{[14]}$. Extra renal lesions are present in 35\% of patients and can cause clinical symptoms, and extra renal LC deposition has a clear, independent effect on patient survival ${ }^{[10]}$.

LCDD is frequently misdiagnosed as a protein disease. Up to $50 \%$ of patients are diagnosed with LCDD secondary to $\mathrm{MM}$ or other lymphoproliferative diseases, in the remaining patients, LCDD occurs singly, as in our patient. However, an unusual case with LCDD, renal amyloidosis and cast nephropathy has been described ${ }^{[11]}$. Other associations seen in a series of 24 cases were with cast nephropathy with light chain disease and light chain disease with amyloidosis of other organs (tongue and heart) and LCDD has also been known to occur in conjunction with heavy chain disease ${ }^{[12]}$. The diagnosis of LCDD can be made with a kidney biopsy. The characteristic morphological findings in LCDD are nodular glomerulosclerosis and nonfibrillar electron-dense deposits on the glomerular or tubular basement membrane, as seen with electron microscopy (EM). Most patients with LCDD and nephrotic syndrome have or rapidly develop renal dysfunction (as in our patient), renal function declines rapidly as a rapidly progressive glomerulonephritis or acute tubulointerstitial nephritis [4]. However, the patients may present with proteinuria in the absence of overt renal dysfunction ${ }^{[13]}$, and with asymptomatic proteinuria ${ }^{[11]}$.

The factors independently associated with a worse renal prognosis are age (relative risk [RR] 1.05; 95\% confidence interval $[\mathrm{CI}] 1.009$ to 1.086 ) and serum creatinine at 
presentation (RR, 1.24; 95\% CI, 1.02 to 1.5). Those independently associated with a worse patient survival are age (RR, $1.06 ; 95 \% \mathrm{CI}, 1.03$ to 1.1$), \mathrm{MM}$ (RR, 2.75; $95 \%$ CI, 1.22 to 6.2), and extra renal LC deposition (RR, 2.24; 95\% CI, 1.15 to 4.35 ). While kappa-LC deposition is more frequently associated with nodular sclerosing glomerulopathy, histological parameters are not predictors of renal/patient prognosis. The survival of the uremic patients undergoing dialysis is similar to that of patients not reaching uremia ${ }^{[10]}$. Our patient did survive for 4 years on HD, and died due to Covid 19 pneumonia.

\section{Conclusion and clinical pearls}

Proteinuria should not be ignored. A patient having long standing hypertension who develops proteinuria should be investigated, as there may be other cause as it happened in our patient. If a kidney biopsy was done earlier, LCDD could have been diagnosed at a time with normal renal function, and avoiding dialysis. Patient's dialysis dependency and the associated problems related to his vascular access prevented him from undergoing ASCT.

\section{References}

1. Merlini G, Wechalekar AD, Palladini G. Systemic light chain amyloidosis: an update for treating physicians. Blood. 2013;121(26):5124-30.

2. Rosenbaum C, Jasielec J, Laubach J, Paba Prada C, Richardson P, Jakubowiak AJ. Evolving strategies in the initial treatment of multiple myeloma. Semin Oncol. 2013;40(5):592-601.

3. Bridoux F, Desport E, Frémeaux-Bacchi V, Chong CF, Gombert JM, Lacombe C, et al. Glomerulonephritis with isolated $\mathrm{C} 3$ deposits and monoclonal gammopathy: a fortuitous association? Clin J Am Soc Nephrol. 2011;6(9):2165-74.

4. McKenna RW, Kyle RA, Kuehl WM, Grogan TM, Harris NL, Coupland RW. Plasma Cell Neoplasms in WHO Classification of Tumours of Haematopoietic and Lymphoid Tissues. Lyon: International Agency for Research on Cancer. 2008.

5. Levey AS, Eckardt K, Dorman NM, et al. Nomenclature for kidney function and disease: report of a Kidney Disease: Improving Global Outcomes (KDIGO) Consensus Conference. Kidney Int. 2020;97:1117-29.

6. Sayed RH, Wechalekar AD, Gilbertson JA, et al. Natural history and outcome of light chain deposition disease. Blood. 2015;126(26):2805-10.

7. Jimenez-Zepeda VH. Light chain deposition disease: novel biological insights and treatment advances. Int J Lab Hematol. 2012;34:347-55.

8. Randall RE, Williamson WC Jr, Mullinax F, Tung MY, Still WJ. Manifestations of systemic light chain deposition. Am J Med. 1976;60:293-99.

9. Sukmawati Tansil Tan, Yohanes Firmansyah, Jessica Elizabeth. New approach to skin burn treatment: Potential of secretome from Wharton's jelly mesenchymal stem cell therapy. Int. J Adv. Biochem. Res. 2020;4(2):11-16.

DOI: 10.33545/26174693.2020.v4.i2a.49

10. Pozzi C, D'Amico M, Fogazzi GB, et al. Light chain deposition disease with renal involvement: clinical characteristics and prognostic factors. Am J Kidney Dis. 2003;42(6):1154-63.
11. Salant DJ, Sanchorawala V, D'Agati VD. A Case of Atypical Light Chain Deposition Disease-Diagnosis and Treatment. Clin J Am Soc Nephrol. 2007;2(4):85867.

12. Pant AD, Solez K. Light chain deposition disease in kidney: A review of the literature. J Pathol Nepal. 2011;1:56-9.

13. Buxbaum JN, Chuba JV, Hellman GC, Solomon A, Gallo GR. Monoclonal immunoglobulin deposition disease: Light chain and light and heavy chain deposition diseases and their relation to light chain amyloidosis. Clinical features, immunopathology, and molecular analysis. Ann Intern Med. 1990;112:455-64.

14. Qian Q, Leung N, Theis JD, Dogan A, Sethi S. Coexistence of myeloma cast nephropathy, light chain deposition disease, and nonamyloid fibrils in a patient with multiple myeloma. Am J Kidney Dis. 2010;56(5):971-76. 